\title{
Eagle syndrome: from neck pain to vagal episode: report of two cases
}

\author{
Robin Jouan ${ }^{1, *}$, Frédéric Faure ${ }^{2}$, Olivier Robin ${ }^{3}$ \\ ${ }^{1}$ Oral surgeon, Departement of Maxillofacial Surgery, Centre Hospitalier de Villefranche sur Saone, 69400Gleize, France \\ 2 Hospital Practitioner, ENT and Cervicofacial Surgery, Hospices Civils de Lyon, Lyon 69008, France \\ 3 University Professor, Hospital Practitioner, Odontology Department, Hospices Civils de Lyon, Lyon 69007, France \\ * Correspondence: jouan.robin@gmail.com
}

Keywords:

eagle syndrome / styloid / piezosurgery / orofacial pain

(Received: 14 November 2016, accepted: 1 February 2017)

\begin{abstract}
Purpose: Eagle syndrome is a rare symptomatic clinical and radiological entity. It is characterized by an elongation of the styloid process or calcification of the stylohyoid ligament, and it typically causes head and neck pain, odynophagia, otalgia, or headache. Observation: The first case dealt with an elongation of the left styloid by $46 \mathrm{~mm}$, and the second case dealt with a calcification of the right stylohyoid ligament at the lesser horn of the hyoid bone. In both cases, cervical rotation caused a vagal episode by the compression of the carotid arteries. Both cases were treated surgically, the first with an intrabuccal procedure and the second by cervicotomy. Both patients have shown no postoperative recurrence of their symptoms. Conclusion: Eagle syndrome, although rare, should be considered when patients describe pain symptoms with no identified etiology in the head and neck area. A simple additional diagnostic procedure such as a computed tomography (CT) scan or an orthopantomogram can easily show a long styloid process or calcified stylohyoid ligament and their anatomical relations, in particular, any vascular relations. The use of piezosurgery decreases intraoperative risks in the mastoid cut of styloid process.
\end{abstract}

\section{Introduction}

Described for the first time by Eagle in 1937 [1], Eagle syndrome consists of a set of symptoms of the head and neck region. Its etiology is the calcification of the stylohyoid ligament or elongation of the styloid process over the average length of $25 \mathrm{~mm}$, and it affects approximately $5 \%$ of the population [2].

The classical clinical presentation is a unilateral oropharyngeal pain, exacerbated by swallowing, tonsillar fossa palpation, or cephalic torsion [2]. Some patients associate feelings of dizziness, otalgia, headache, or even brief loss of consciousness when the styloid is in a posterior or lateral position [3]. Here we present two clinical cases: the first is a typical elongation of the styloid process and the second is an ossification of the lower segment of the stylohyoid ligament, in level with the lesser horn of the hyoid bone. These two cases were successfully treated surgically.

\section{Observation}

\section{Case 1}

The case was a 31-year-old patient without significant medical or surgical history. The patient presented with typical pains in the left oropharyngeal region exacerbated by tonsillar fossa palpation and swallowing along with recurrent episodes of vagal discomfort.

The computed tomography (CT) scan showed a left styloid process of length $46 \mathrm{~mm}$ versus $22.4 \mathrm{~mm}$ on the right (Fig. 1ac). An intraoral surgical section was planned (Fig. 2a-d).

The procedure was performed under general anesthesia, in a supine position with nasotracheal intubation through the right nostril. The procedure began with a left tonsillectomy that exposed the floor of the tonsillar fossa, thus facilitating access to the styloid process, which was performed using a rigid endoscope set at $0^{\circ}$. The styloid process and its prolongation had been exposed from the mastoid tip to the stylohyoid ligament. The styloid process was resected by piezosurgery to decrease the risk of vascular injury. The stylohyoid ligament was incised with scissors to detach it from the styloid and was not reattached.

\section{Case 2}

A 44-year-old woman presented with pain on the right side of the face for 5 months without identifiable triggers. She described otalgia on the right side, temporal and periorbital pain, dizziness, and syncope, which was induced with right cephalic torsion.

CT and magnetic resonance imaging (MRI), showed a $27 \mathrm{~mm}$ calcification of the stylohyoid ligament (Fig. 3) in its lower part 


\section{SH ORT CASE REPORT}

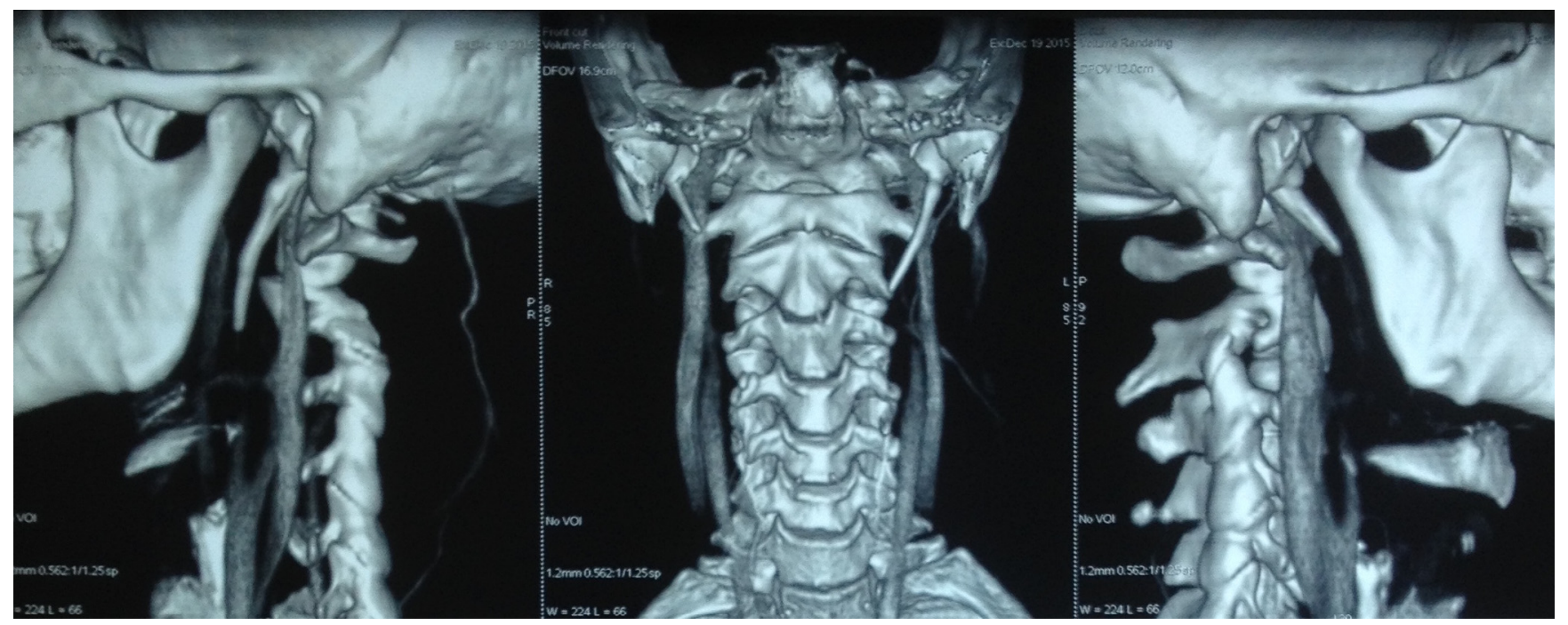

Fig. 1. (a) CT 3D reconstruction showing a 46-mm-long left styloid process. (b) Anteroposterior view. (c) Contralateral styloid process.

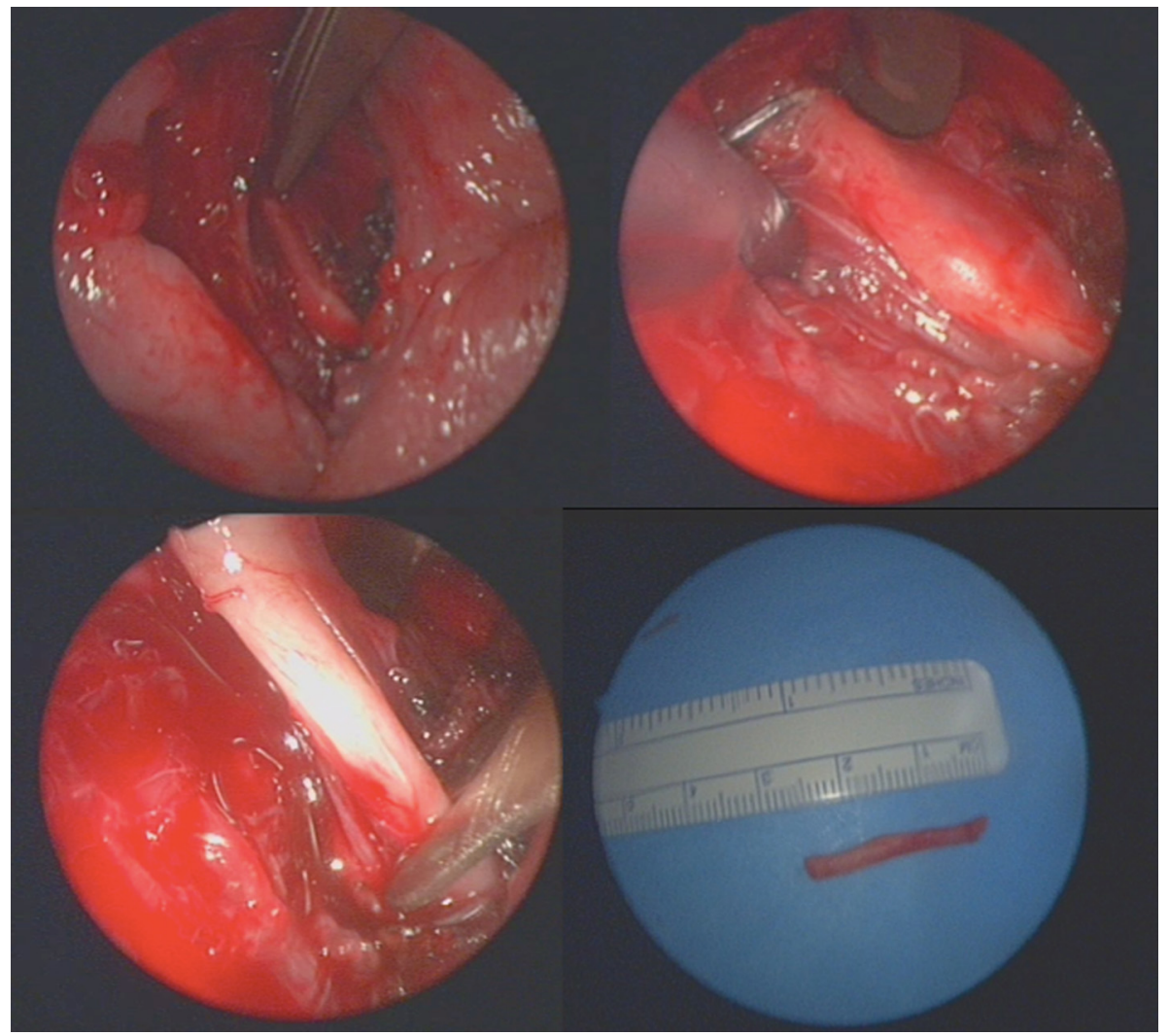

Fig. 2. (a) Endoscopic view after tonsillectomy. (b) Endoscopic view of the styloid process. (c) Endoscopic view showing the resected stylohyoid ligament. (d) The resected styloid process.

at the level of the lesser horn of the hyoid bone. A surgical resection of the calcified segment was performed.

The procedure was performed under general anesthesia, in a supine position, with orotracheal intubation. An anterior approach through a Sébileau-type incision was used and dissection of the carotid-jugular axis in the direction the carotid bulb. The calcified lower part of the stylohyoid ligament and the carotid artery were observed to be in close proximity. Thus, the stylohyoid ligament resection and the removal of part of the hyoid bone were performed. 


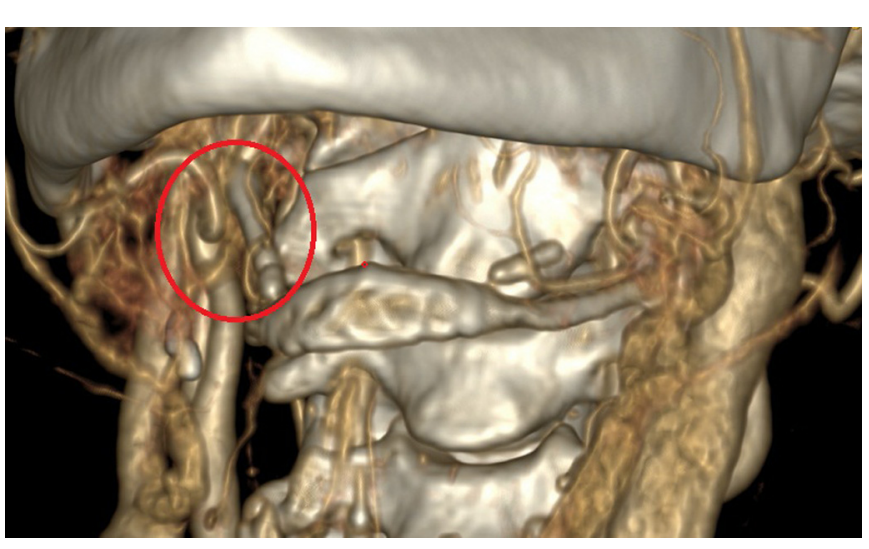

Fig. 3. 3D-CT reconstruction showing calcification of the stylohyoid ligament and the proximity to the carotid artery.

\section{Comments}

Eagle syndrome was described in 1937 by Eagle. Initially, the diagnosis could only be made using an orthopantomogram [4]. Whether or not the length of the styloid process is responsible for symptomatology has been debated because some patients are asymptomatic when the length exceeds the average $(25 \mathrm{~mm})$. In the presence of a clinical symptomatology, including orofacial and neck pains as in the two cases described, the use of an contrast-enhanced CT is interesting because it allows the confirmation of the proximity between calcification and vascular structures, including the carotid artery.

The radiological examination is a valuable aid to help us choose which treatment to carry out (intraoral surgery or cervicotomy). In the first case, the medial and posterior location of the styloid process, as well as its palpability in an intraoral examination, led us to choose an intraoral approach. The use of rigid endoscope set at $0^{\circ}$ optimized the path through the tonsillar fossa and the dissection around the styloid process. This route first helps to avoid cutaneous scarring, although it is associated with a greater risk of infectious complications [5]. In the second case, cervical localization led us to use an anterior cervical approach. The use of the piezoelectric system to resect the styloid process as high as possible helped to perform the surgery but avoid vascular injuries. Alternatives include the use of an osteotome, which has a greater risk of injury to adjacent structures without the benefit of the efficiency or operator ease of use, or the use of a bone gouge, which is less accurate than the piezotome [5].

Therefore, we propose to extend the use of the injected $\mathrm{CT}$ with three-dimensional reconstruction for the diagnosis of Eagle syndrome. Using computed tomography alone or cone-beam CT, it's impossible to visualize proximity of the major vessels. However visualize vascular axes is needed for adequate surgical planning. Similarly, the orthopantomogram is a contributory examination when diagnosing, but is insufficient for planning surgery.

These two patients, who were followed up at 15 days and 2 months after surgery, did not have any sequelae or recurrences of their symptoms.

\section{Conflicts of interests}

The authors declare that they have no conflicts of interest in relation to this article.

\section{References}

1. Eagle WW. Elongated styloid processes. Report of two cases. Arch Otolaryngol 1937; 25: 584-586.

2. Dulguerov $P$, Kolher R, Becker M. Carotidynie et syndrome d'Eagle: deux syndromes classiques à redécouvrir. Rev Med Suisse 2011; 7: 1929-1934.

3. Bizet A, Margottin C, Lagarde A, Malard 0, Corre P, Lesclous P. Prise en charge chirurgicale par voie endobuccale d'une patiente ateinte d'un syndrome d'Eagle: cas clinique et revue de la littérature. Med Buc Chir Buc 2016; 22: 63-75.

4. Bagga MB, Kumar CA, Yeluri G. Clinicoradiologic evaluation of styloid process calcification. Imaging Sci Dent 2012; 42: 155-161.

5. Sudra Y, Teitelbaum J, Antoine L, Mondié JM, Baudet-Pommel M. Syndrome d'Eagle: à propos d'un cas avec calcifications multiples. Med Buc Chir Buc 2008; 14: 97-102.

Med Buccale Chir Buccale 2017;23:169-171

(C) The authors, 2017

This is an Open Access article distributed under the terms of the Creative Commons Attribution License (http://creativecommons.org/licenses/by/4.0), which permits unrestricted use, distribution, and reproduction in any medium, provided the original work is properly cited. 